\title{
Fibrous pleural tumour producing 171 litres of transudate
}

\author{
C.S. Ulrik, K. Viskum
}

Fibrous pleural tumour producing 171 litres of transudate. C.S. Ulrik, K. Viskum. OERS Journals Ltd.

ABSTRACT: Localized fibrous tumours of the pleura (or localized benign mesothelioma) are rare, and in most cases, asymptomatic.

This report describes a $48 \mathrm{yr}$ old female with a right-sided fibrous pleural tumour, which produced $171 \mathrm{~L}$ of transudate before a correct diagnosis was reached. The tumour was surgically removed and the transudation stopped immediately.

Eur Respir J 1998; 12: 1230-1232.
Respiratory Medicine Section, RHIMA Center, Rigshospitalet, Copenhagen, Denmark.

Correspondence: C.S. Ulrik, Respiratory Medicine Section ML 7721, RHIMA Center, Rigshospitalet, DK-2200 Copenhagen N, Denmark, Fax: 4535456648

Keywords: Benign mesothelioma, localized fibrous tumour, pleura, transudate

Received: February 51998

Accepted after revision April 11998
Localized fibrous tumour, also called benign mesothelioma [1], of the pleura is a rare tumour that in approximately $10 \%$ of the cases is associated with a pleural effusion $[2,3]$. We report the case of a 48 yr old female with a benign pleural fibrous mesothelioma, that produced $171 \mathrm{~L}$ of transudate before the patient was cured by radical excision of the tumour.

\section{Case report}

\section{History}

In October 1996, a 48 yr old, nonsmoking female with no history of alcohol or drug misuse was admitted to a department of pulmonary medicine owing to shortness of breath of 2 weeks' duration.

For 6 months prior to the admission she had had a slight pain in her right shoulder, but apart from that she had been well. She had used no medication other than acetaminophen, codeine and ibuprofen, and had no known allergy. At the age of $1 \mathrm{yr}$ she had been admitted to hospital because of tuberculosis, but no records concerning diagnosis or treatment were available. For years she had worked as a laboratory technician, specializing in culturing Aspergillus niger, with no work-related symptoms. A fibroma of the uterus had been diagnosed 2 yrs previously, and she had been offered a hysterectomy whenever convenient.

\section{Findings on admission}

At the time of admission, the chest radiograph revealed a cyst in the right upper lobe and a shadowing of the right hemithorax with mediastinal shift to the left compatible with a large, right-sided pleural effusion. A computed tomography (CT) scan performed shortly afterwards was described as follows: right-sided pleural effusion, consolidation of the right lower lobe and a $3 \mathrm{~cm}$ large cyst in the upper lobe of the right lung.
Routine blood analyses, including glucose, erythrocyte sedimentation rate (ESR), differential leukocyte count, amylase, lactate dehydrogenase (LDH), alkaline phosphatase and albumin, were within the reference limits, and no traces of sugar, blood or protein were present in the urine.

The patient had no history or signs of heart, liver, kidney or thyroid disease. The electrocardiogram (ECG) was without signs of cor pulmonale, and an echocardiography was described as normal apart from a slight tricuspid regurgitation (maximum gradient $30-40 \mathrm{mmHg}$ ) and especially no dilatation of the right ventricle.

\section{Initial thoracocentesis and thoracoscopy}

A right-sided thoracocentesis was performed, and $3.3 \mathrm{~L}$ of yellow, translucent fluid was evacuated. The fluid contained $13 \mathrm{~g} \cdot \mathrm{L}^{-1}$ of protein, and cytology showed reactive mesothelial cells without suspicion of malignancy. The transudate recurred within days.

A thoracoscopy was performed, revealing an atelectatic lower lobe and a moderate irritation of the parietal pleura with vascular injection and no fibrin deposits. Parietal biopsies showed an essentially normal pleura. Biopsies were also cultured for mycobacteria (negative, reported later).

The pleural fluid was tested using the polymerase chain reaction (PCR) technique and, whenever possible, cultured for virus (adenovirus, enterovirus, cytomegalovirus, herpes simplex virus and Varicella-zoster virus), parasites and bacteria, including mycobacteria. All tests were negative. Blood radioallergosorbent tests for Aspergillus niger and A. fumigatus were also negative.

\section{Continued transudation and repeated thoracocenteses}

The ESR increased to $85 \mathrm{~mm}$ and the plasma albumin gradually dropped to $460 \mu \mathrm{M}$. All other parameters remained within reference limits, including antinuclear cytoplasmic 
antibody (ANCA) and antinuclear antibodies (ANA) (immunoglobulin $\mathrm{G})$.

The patient suffered much discomfort due to the continuing production of approximately $5 \mathrm{~L}$ of pleural fluid per week. Before each thoracocentesis, which was performed at least twice a week, she was short of breath. At no time during her illness did she have symptoms of hypoglycaemia or osteoarthropathy and the temperature remained normal. Approximately 6 weeks after admission, the evacuated pleural fluid showed eosinophilia, which was not found in the peripheral blood.

\section{Further diagnostic procedures}

Two months after admission, another thoracoscopy was performed. The vascular injection of the parietal pleura was more pronounced and a few fibrin deposits were seen. Biopsies from both parietal and visceral pleura showed severe, nonspecific pleuritis with eosinophilia, and no changes compatible with tuberculosis or malignancy. The underlying disease causing the continuous production of huge amounts of transudate was still a mystery.

The patient considered occupational exposure and sensitization to Aspergillus to be a serious candidate as the underlying cause. Although no clinical findings, possibly apart from a high number of eosinophils in the pleural fluid, supported this theory, she was given a two-week course of prednisolone (30 mg.day-1). No change in her condition was observed.

Two months after admission, an ultrasound examination of the abdomen confirmed the presence of a partially calcified $10 \times 10 \times 7 \mathrm{~cm}$ large mass in the uterine wall. No ascites or abnormalities in the ovaries were observed. As no explanation for the transudation had been found, the possibility of pseudo-Meigs' syndrome was considered and a hysterectomy was performed in January 1997. Apart from a $8 \times 8 \times 8 \mathrm{~cm}$ large fibroma, nothing abnormal was found in the abdomen. Histology showed a partially calcified leiomyoma. The formation of transudate continued.

Five months after admission, a ventilation-perfusion

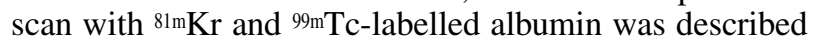
with sequelae after right-sided pleural effusion. Part of the right lower lobe showed ventilation but no perfusion.

Six months after admission, the biochemistry of the pleural fluid showed: Na $144 \mathrm{mM}$, K $3.7 \mathrm{mM}$, creatinine $0.061 \mathrm{mM}$, glucose $6.1 \mathrm{mM}$, amylase $10 \mathrm{U} \cdot \mathrm{L}^{-1}$, LDH 85 $\mathrm{U} \cdot \mathrm{L}^{-1}$, triglyceride not detectable, alkaline phosphatase 12 $\mathrm{U} \cdot \mathrm{L}^{-1}$, and total protein $9 \mathrm{~g} \cdot \mathrm{L}^{-1}$, i.e. all values within the reference range and giving no clue to the aetiology, but confirming the effusion being a transudate. So far, the pleural fluid had been examined several times, showing an increasing leukocyte count, especially a high number of eosinophils, and an abundance of proliferating mesothelial cells. No micro-organisms or parasites were identified.

The CT scan was repeated 6 months after admission, this time as a high-resolution scan, and was described as follows. A solid inhomogeneous infiltrate measuring $9 \times 9 \times 6 \mathrm{~cm}$ was seen in the posterior basal part of the right pleural cavity. No connection to an essentially normal lung was seen and there was no fluid in the right pleural cavity. The cyst in the right upper lobe was unchanged, and the infiltrate was free of the aorta and the inferior vena cava.

\section{Final operation and diagnosis}

A thoracotomy was performed and a tumour measuring $20 \times 20 \times 7 \mathrm{~cm}$ was removed. It was partially adherent to the lower lobe of the lung and macroscopically had no bronchial connection; the blood supply came through the diaphragm. The tumour was separated from the lung and diaphragm by electrocoagulation and a partial decortication was performed. The surgeons judged the tumour as consisting of pulmonary tissue and, in agreement with this, the perioperative histology noted pulmonary sequester.

The production of pleural fluid stopped immediately after the removal of the tumour. During the course of the disease a total of $171 \mathrm{~L}$ of pleural fluid had been removed.

The final histological examination showed a mesenchymal tumour with spindle cell structure and no organ specific findings. The central part of the tumour consisted of relatively large irregular arteries with a thickened intima mixed with thin-walled vessels. Between these findings, areas with closely packed tumour cells were seen, occasionally in a herring-bone pattern. The mitotic activity was low. Morphologically the differential diagnosis was between benign haemangiopericytoma and benign fibrous tumour, also called localized fibrous mesothelioma. Immunohistochemically, the tumour cells were positive for vimentin and negative for two cytokeratins, confirming the latter diagnosis to be correct.

The patient has been well ever since.

\section{Discussion}

The extended course of the disease in this patient was caused by misinterpretation of the first CT scan and of two thoracoscopies. The tumour must have been seen on both thoracoscopies but misinterpreted as part of the lower lobe of the lung. The effusion was a transudate with a persistently low content of protein.

Clinically, the patient had no signs of heart, liver, kidney or thyroid disease or pulmonary embolism, the most common causes for transudative effusions [1]. The blood chemical findings, ECG, echocardiography and ventilationperfusion scan did not reveal any signs of diseases in these organs. Obstruction of the veins from the right lung was considered as a possible cause but was not detected by CT scan, echocardiography or ventilation-perfusion scintigraphy.

A number of causes which in most cases lead to an exudative effusion was considered in desperation. No underlying microbiological or parasitic cause was found and no evidence of connective tissue disease or vasculitis was found. An allergic reaction induced by occupational exposure to A. niger could not be confirmed. The increasing number of eosinophils in the pleural fluid was probably caused by the repeated thoracocenteses [1].

The patient had not taken medication known to induce pleural effusions. The criteria for a diagnosis of Meigs syndrome [4] were not fulfilled; however, the fibroma of the uterus was considered as a possible cause [5, 6] and the patient therefore had a hysterectomy, which did not in any way influence the production of pleural fluid.

Finally, a high-resolution CT scan was performed. The description this time indicated the probable presence of a 
pleural tumour. At thoracotomy, a tumour, peroperatively interpreted as a pulmonary sequester, was removed. Subsequent histological studies, however, changed this diagnosis to a benign fibrous mesothelioma $[3,7,8]$.

Fluid production is seen in only $10 \%$ of the patients with this type of tumour [2,3], and this fluid may be a transudative effusion [9].

\section{References}

1. Light RW. Pleural Diseases, 3rd Edn. Baltimore, MD, Williams and Wilkins, 1995.

2. Briselli M, Mark EJ, Dickersin GR. Solitary fibrous tumours of the pleura. Eight new cases and review of 360 cases in the literature. Cancer 1981; 47: 2678-2689.

3. England DM, Hochholzer L, McCarthy MJ. Localized benign and malignant fibrous tumours of the pleura. Am J Surg Pathol 1989; 13: 640-658.
4. Gianoutsos P, Laverty CR. Uterine fibroid: an unusual cause of recurrent haemorrhagic pleural effusion. Med $J$ Aust 1975; 2: 600-602.

5. Meigs JV, Cass JW. Fibroma of the ovary with ascites and hydrothorax. Am J Obstet Gynecol 1937; 33: 249-267.

6. Meigs JV. Pelvic tumours other than fibromas of the ovary with ascites and hydrothorax. Obstet Gynecol 1954; 3: 471-486.

7. Azumi N, Battifora H. Distribution of vimentin and keratin in epithelial and nonepithelial neoplasms: an immunohistochemical study of formalin- and alcohol- fixed tumours. Lab Invest 1987; 56: 4A.

8. Al-Izzi M, Thurlow NP, Corrin B. Pleural mesothelioma of connective tissue type, localized fibrous tumour of the pleura, and reactive submesothelial hyperplasia. An immunohistochemical comparison. J Pathol 1989; 158: 41-44.

9. Hansen RM, Caya JG, Clowry LJ, et al. Benign mesothelial proliferation with effusion. Am J Med 1984; 77: 887892. 